\title{
The Higgs particle and the lattice
}

\author{
Julius Kuti* \\ Department of Physics 0319, University of California, San Diego \\ 9500 Gilman Drive, La Jolla, CA 92093, USA \\ E-mail: jkuti@ucsd.edu
}

\begin{abstract}
After its energy upgrade the Large Hadron Collider (LHC) will directly look for new physics in the LHC14 run including searches for supersymmetry (SUSY) and Beyond the Standard Model (BSM) signals of the composite Higgs mechanism as alternates to the elementary Higgs of the Standard Model. Recent non-perturbative BSM lattice studies is the main focus of this talk. We will demonstrate that non-perturbative simulations of strongly coupled gauge theories (SCGT) have predictive power for experimental searches on the Energy Frontier. Lattice simulations can significantly contribute to our understanding of important BSM paradigms, like SUSY, conformal and near-conformal quantum field theories with implications for the dilaton state, or the alternative composite light scalar as the scalar pseudo-Goldstone boson from global symmetry breaking. Exploratory lattice studies identify tantalizing candidate theories for the composite Higgs mechanism with a light scalar state (Higgs impostor) on the Electroweak scale. The viability of this paradigm requires a composite resonance spectrum in the $2 \mathrm{TeV}$ range, or higher, far separated from the Electroweak scale, and perhaps within the reach of the LHC14 run. The written version of the talk closely follows the material presented at the conference without reviewing postconference developments in the field. Detailed reporting in this plenary talk had to be selective in the alloted time and space without full and comprehensive review of every interesting aspect of the parallel BSM sessions.
\end{abstract}

31st International Symposium on Lattice Field Theory - LATTICE 2013

July 29 - August 3, 2013

Mainz, Germany

\footnotetext{
*Invited plenary speaker at the conference.
} 


\section{Introduction}

The recent discovery of the Higgs boson at $126 \mathrm{GeV}$ by the CMS [1] and ATLAS [2] experiments at the LHC provides the first insight into the origin of Electroweak symmetry breaking (EWSB) in the Standard Model (SM). The minimal realization of EWSB is implemented by introducing an $\mathrm{SU}(2)$ doublet Higgs field as an elementary scalar whose vacuum expectation value (VEV), with $\mathrm{VEV}=246 \mathrm{GeV}$, sets the Electroweak scale. This simple description in terms of an elementary Higgs field is viewed by most theorists as a parametrization rather than a dynamical explanation of EWSB. In particular, without new BSM physics on the TeV scale, the mass-squared parameter of the light and elementary Higgs has to be finely tuned, leading to the well-known hierarchy problem.

Measured properties of the Higgs particle are not precise enough to know if the discovery will validate the SM to energy scales far beyond what can be reached in the forseeable future, or new BSM physics will have to emerge within the reach of the next LHC14 run starting in 2015. After the energy upgrade, LHC will directly look for new physics including searches for SUSY and BSM signals of the light Higgs from compositeness as alternate explanations to the elmentary scalar field in the SM description. Contributions to the new phase of the LHC program is one of the high priority missions of the lattice community. Simulation results from strongly coupled gauge theories, like the ones discussed here, also contribute to our theoretical understanding in a broader sense including conformal and near-conformal gauge theories and important non-perturbative aspects of supersymmetry. As an example, the spontaneous symmetry breaking of scale invariance with its pseudo-Goldstone dilaton state close to the conformal window has been the focus of several recent studies in connection with the Higgs discovery.

Searching for a deeper dynamical explanation and trying to resolve the shortcomings of the elementary Higgs doublet, new BSM physics of strongly-interacting gauge theories was outside experimental reach and without powerful non-perturbative lattice tools when it was first introduced [3-10]. The original framework has been considerably extended by new explorations of the multidimensional theory space in fermion flavor number, the choice of color gauge group, and fermion representation [11-20]. Systematic and non-perturbative lattice studies play an important role in studies of this extended theory space [21-62] with a large number of new contributions presented in BSM sessions at the conference. In the time and space alloted I will discuss some of the contributions and related recent publications.

Of course we hear voices that pursuing the composite Higgs scenario is overtaken by recent findings at the LHC. After all, a light Higgs-like scalar was found, consistent with SM predictions within errors, and composite states have not been found below the TeV scale. In contrast, the voices continue, strongly coupled BSM gauge theories are Higgs-less with resonances predicted below the $\mathrm{TeV}$ scale. Facts do not support this dismissive view which originates from naively scaled up properties of Quantum Chromodynamics (QCD) and related old technicolor guessing games lacking predictive power close to the conformal window where gauge theories are nearly scale invariant, in sharp contrast to QCD which is not. In fact, it has not been shown that compositeness and a light Higgs scalar are incompatible. I will discuss recent developments with conference contributions hinting perhaps the opposite. For example, in near-conformal gauge theories a light composite scalar can emerge on the Electroweak scale with a resonance spectrum far separated 
above the TeV scale, perhaps within the reach of LHC14.

Section 2 is a brief overview of the BSM parallel sessions with apologies for incompleteness. In Section 3 I will briefly discuss lattice tests of the conformal window and the related infrared fixed point of the beta function. I will illustrate this with two models which have been investigated extensively using lattice tools in search for their conformal properties. The main topic of Section 4 is the emergence of light scalars in near-conformal gauge theories. In Section 5 I will briefly review the idea and lattice implementation of the light Higgs as a scalar pseudo-Goldstone boson. Section 6 is a short summary of recent lattice developments of SUSY. Section 7 illustrates lattice applications of BSM phenomenology.

\section{Overview of the BSM parallel sessions}

The main interest has been to investigate strongly coupled gauge theories (SCGT) close to the conformal window $(\mathrm{CW})$ with near-conformality, or conformality with exact scale invariance inside the CW. Approximate scale invariance in the near-conformal scenario makes the models very different from naively scaled QCD, hence the great interest. The largest number of parallel contributions reported new results in the SCGT theory space as shown in Figure 1. This navigation map was introduced in [19]. Fourteen models were discussed for $\mathrm{N}=2,3,4$ colors in several fermion representations of the color gauge group, with variations on the number of fermion flavors. Fermions in the fundamental representation of the SU(3) color gauge group were reported at

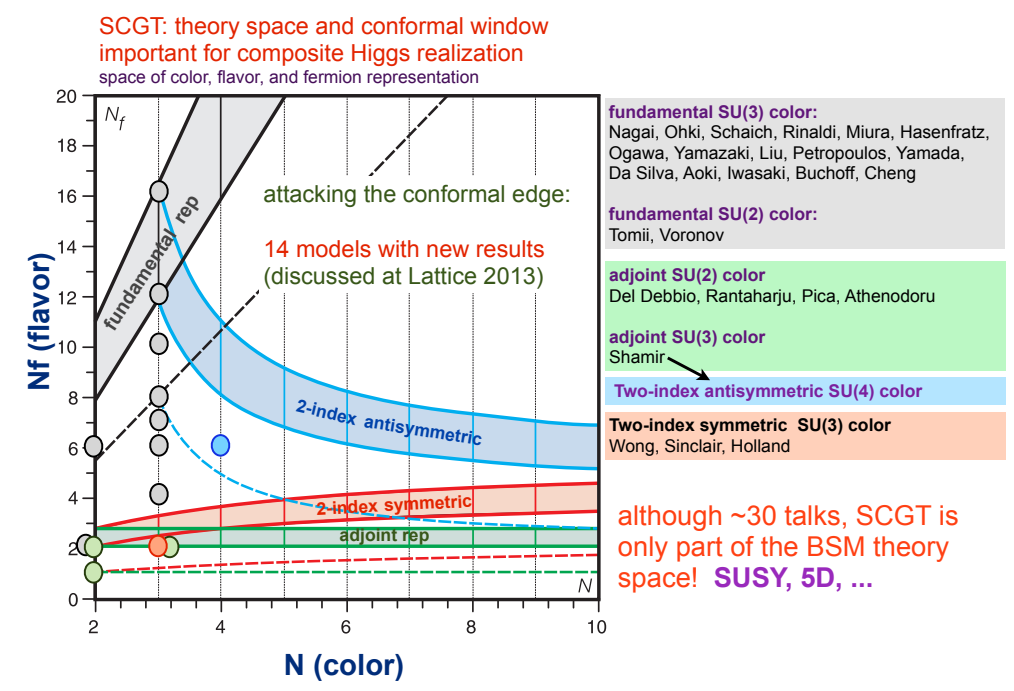

Figure 1: The conformal window for $\mathrm{SU}\left(\mathrm{N}_{\mathrm{c}}\right)$ gauge theories with $\mathrm{Nf}$ fermions in various representations [19]. The shaded regions are the windows, for fundamental (gray), 2-index antisymmetric (blue), 2-index symmetric (red) and adjoint (green) representations in Schwinger-Dyson approximation. The dashed lines indicate earlier expectations for larger conformal windows which were based on the fixed points of two-loop beta functions. Circles mark models with new results reported at the conference in an effort to establish exact classification.

the conference [63-70] for a large range of fermion flavors in and out of the CW. Similar studies in the fundamental representation with the SU(2) gauge group were also reported with interesting results for six flavors [71,72] (the model with broken chiral symmetry would exhibit scalar pseudo-Goldstone bosons). Adjoint representations with SU(2) and SU(3) color gauge group were 
addressed in [73-76]. The two-index antisymmetric representation with 6 flavors and the SU(4) gauge group was reported for the first time [76]. The two-index symmetric representation with 2 flavors and SU(3) gauge group was discussed in three talks [77-79] with the emergence of a light scalar close to the $\mathrm{CW}$. Some results in a more exotic representation with $\mathrm{SO}(4)$ gauge group were also reported at the conference [80].

Before we get bored with the restricted theory space of Figure 1 which will be my primary focus in the talk, other interesting contributions at the conference reminded us that the scope of the theory space can be extended significantly in the BSM paradigm. This includes SUSY with conference contributions [81-84] and 4+1 dimensional Kaluza-Klein and Gauge-Higgs unification [85-88] which some reference as the Hosotani-mechanism. I illustrate some highlights of $4+1 \mathrm{dim}$ results in Figure 2. Other interesting new lattice ideas were also presented including
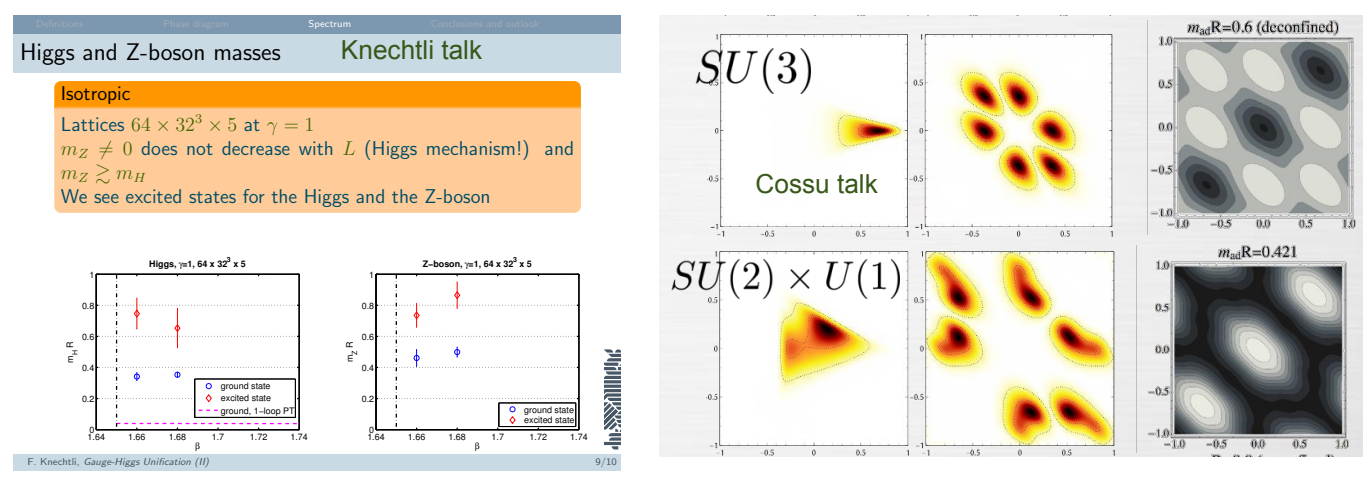

Figure 2: The emergence of the Higgs on the lattice from 5 dimensions [85], and symmetry patterns of Polyakov loop spectra from the Hosotani mechanism of Gauge-Higgs unification [88].

Higgs-Yukawa models with spectroscopy and symmetry breaking patterns [89-92], the role of fourfermion operators [93,94], conformal radial quantization [95], large $\mathrm{N}$ studies [96-100], quantum gravity [101-103], dark matter on the lattice [104], and the early universe on the lattice [104,105].

\section{The conformal window on the lattice}

Strongly coupled gauge theories, with massless fermions in some representation of the SU(N) gauge group, can exhibit infrared fixed points (IRFP) inside the CW with vanishing beta function. The gauge group, flavor, and representation dependent position of the $\mathrm{CW}$ is shown in Figure 1 from the 2-loop perturbative beta function and from Schwingwer-Dyson approximation [19]. Neither approximation is reliable. Lattice efforts aim to establish the exact location of the $\mathrm{CW}$ from first principles and with important BSM model building consequences. The lattice toolsets will be illustrated in two frequently discussed models searching for their conformal behavior. The first model is Minimal Walking Technicolor (MWT) which is defined with two fermion flavors in the adjoint representation of the SU(2) gauge group [17]. The second model has twelve fermion flavors in the fundemantal representation of the SU(3) gauge group with somewhat controversial and inconclusive results. 


\subsection{Two fermion flavors in the adjoint representation of the $\mathrm{SU}(2)$ gauge group}

Minimal Walking Technicolor (MWT) is defined with two massless fermions in the adjoint representation of the $\mathrm{SU}(2)$ gauge group [17].

\section{Running coupling and $\beta$ function:}

The 2-loop beta function, although not reliable at strong coupling, develops a zero with an IRFP inside the $\mathrm{CW}$. It is very difficult to resolve the small and nearly vanishing $\beta$ function of slow walking from the existence of a conformal fixed point where the beta function develops a zero. The main problem is to bridge many orders of magnitude in scale which is neccessary to run the gauge coupling from the UV fixed point toward the far infrared scale controlled by the IRFP. Defined from the gradient flow of the gauge field [108], a new gauge coupling was proposed recently [106,107]. Since the gradient flow probes the gauge field on the scale $\sqrt{8 \mathrm{t}}$, the new running coupling can be defined as a function of $\mathrm{L}$ in finite volume $\mathrm{V}=\mathrm{L}^{4}$ while holding $\mathrm{c}=(8 \mathrm{t})^{1 / 2} / \mathrm{L}$ fixed:

$$
\alpha_{\mathrm{c}}(\mathrm{L})=\frac{4 \pi}{3} \frac{\left\langle\mathrm{t}^{2} \mathrm{E}(\mathrm{t})\right\rangle}{1+\delta(\mathrm{c})}
$$

This volume dependent coupling includes the tree level normalization factor $\delta(\mathrm{c})$ which is known analytically $[106,107]$. The new running coupling scheme provides a very useful alternative to the original Schrödinger functional scheme [109]. The measured renormalized couplings are very
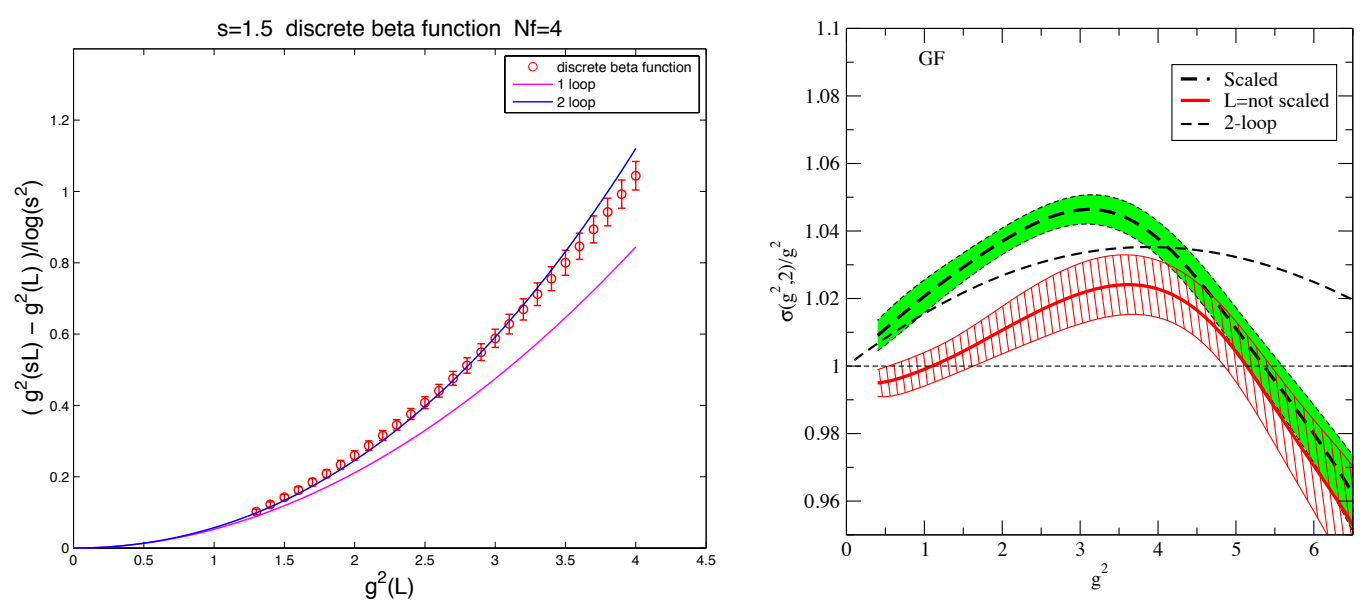

Figure 3: The plot on the left side shows agreement in the continuum limit with the perturbative step function (discrete $\beta$-function) at weak coupling in the $\mathrm{Nf}=4$ test of the new method $[106,107]$. On the right: the MWT application with Schrödinger boundary condition [74]. The black line with the green band and the red line with the hashed error band correspond to two different continuum extrapolations of the step function normalized to $\mathrm{g}^{2}$. The black dashed line gives the 2-loop perturbative result. A clearly identified zero develops in the step function and beta function around $\mathrm{g}^{2} \sim 5$ where the plotted ratio crosses one.

accurate and the scheme defines a one-parameter family which can be adjusted to several goals. It was extensively tested with four massless fermions in the fundamental representation of the SU(3) color gauge group [107] with results shown in Figure 3. In the Nf $=4$ tests antiperiodic boundary conditions were used for massless fermion in all four directions of the finite four-dimensional volume and the boundary condition of the gauge field was kept periodic. The gradient flow coupling 
with Schrödinger functional boundary condition was tested recently $[110,111]$ and effects of the slowly changing topology on the gradient flow couplign were reported [112]. Application to the MWT model with Schrödinger functional boundary condition is shown in Figure 3 as reported at the conference [74]. It was found that the results are in qualitative agreement with the previous studies using the running coupling of the original Schrödinger functional method [109,113]. All findings in the MWT model are consistent with a zero in the step beta function and the existence of the related IRFP. Results on the gradient flow coupling are promising with small statistical errors and better scaling with the volume. The discretization effects can be reduced by the Symanzik improvement program. I expect many more lattice BSM applications from this method.

\section{Anomalous dimension of the $\bar{\psi} \psi$ operator:}

An efficient lattice method to extract the fermion condensate from the eigenvalue density is via the mode number distribution. One calculates the eigenvalues $\lambda$ of the Hermitian Dirac operator $\mathrm{D}^{\dagger} \mathrm{D}+\mathrm{m}^{2}$ and determines how many eigenvalues are below some cut $\mathrm{M}^{2}$, namely

$$
v(\mathrm{M}, \mathrm{m})=\mathrm{V} \int_{-\Lambda}^{\Lambda} \mathrm{d} \lambda \rho(\lambda, \mathrm{m}),
$$

where $\Lambda=\sqrt{\mathrm{M}^{2}-\mathrm{m}^{2}}$. The mode number $v(\mathrm{M}, \mathrm{m})$ is renormalization-group invariant [114, 115] and at leading order one can define an effective fermion condensate $\Sigma_{\text {eff }}=(\pi / 2 \mathrm{~V}) \mathrm{d} v(\mathrm{M}, \mathrm{m}) / \mathrm{d} \Lambda$, which in the chiral limit yields the condensate $\Sigma$ if the chiral symmetry of the theory is broken [115].

The mode number distribution was recently used in the context of BSM lattice gauge theories to extract the anomalous mass dimension in the MWT model [116] and in $\mathrm{SU}(3)$ gauge theories with $\mathrm{Nf}=4,8$ or 12 fermions in the fundamental representation [117]. New results were reported at the conference for all four lattice models $[68,73]$.

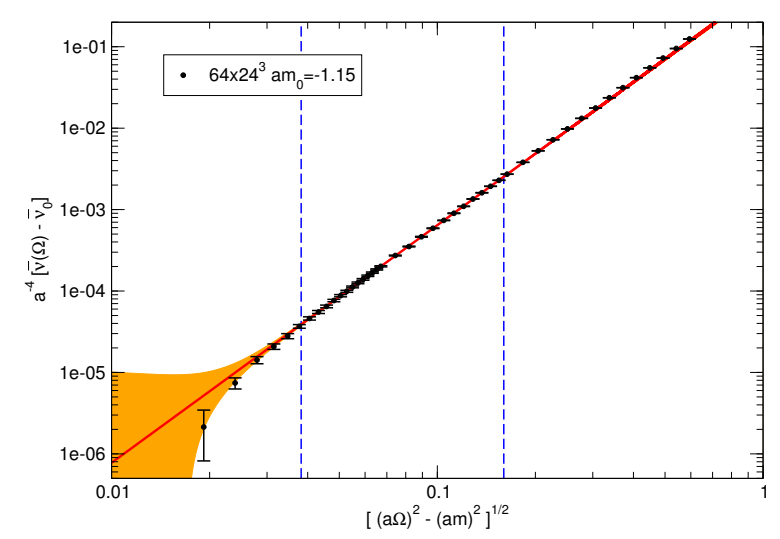

Figure 4: The scale-dependent mode number function of the MWT model is plotted with notation from the original paper [116]. The red line with the hashed error band corresponds to the continuum result at fixed bare gauge coupling $\beta=2.25$. The black dashed line gives the 2-loop perturbative result.

In an IR-conformal gauge theory like MWT, the spectral density and the mode number in some range of the eigenvalues follow power law behavior. The exponent is related to the $\bar{\psi} \psi$ anomalous dimension $\gamma_{*}$ at the IR-fixed point. The mode number function fitted against the eigenvalue scale should be a straight line in a logarithmic plot, as shown in Figure 4 for the MWT model [116]. From the slope of the fit, the anomalous dimension of the MWT model is found to be $\gamma_{*}=0.37(2)$ at fixed bare gauge coupling $\beta=2.25$. This result was confirmed in the new conference contribution [73]. 
Results from the MWT model demonstrate that the mode number method works efficiently in extracting the $\bar{\psi} \psi$ anomalous dimension from BSM lattice simulations.

\section{Mass-deformed spectroscopy and conformal scaling:}

There are two applications of this test working with data extrapolated to infinite volume, or finite volume data analyzed with conformal finite size scaling theory. Working in the infinite volume limit and varying the small fermion mass $\mathrm{m}$ we can determine the fermion mass dependence of the physical masses of composite bound states $\mathrm{M}$ in the Transfer matrix spectrum probing the wellknown relation $\mathrm{M} \sim$ const $\cdot \mathrm{m}^{1 / 1+\gamma_{*}}$ [44]. The exponent $\gamma_{*}$ controls the scaling behavior of $\mathrm{M}$ as a function of small fermion mass deformations around the IRFP. The method requires infinite volume extrapolation from large volume data. In the MWT model approximate volume independence is reached for $\mathrm{M}_{\pi} \cdot \mathrm{L}>10$ for the lowest mass with pseudo-scalar (pion) quantum numbers. Figure 5 depicts the infinite volume extrapolation of a limited part of the spectrum [73].

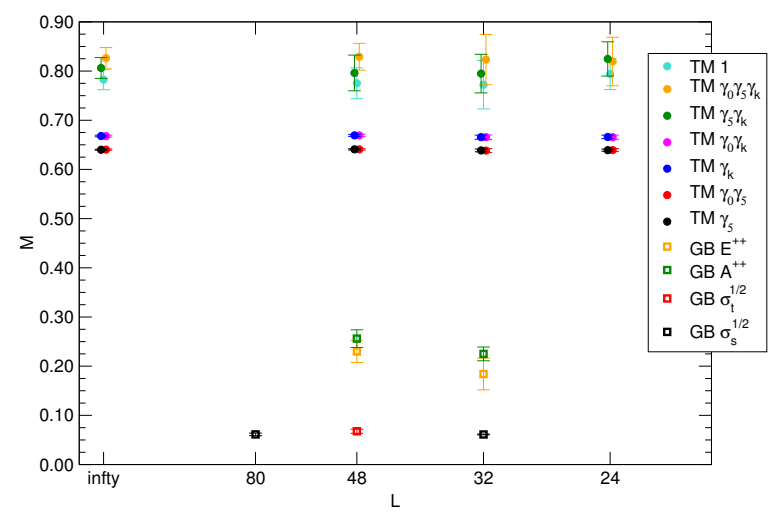

Figure 5: Representative spectrum of mesonic states in the MWT analysis at PCAC fermion mass am $=0.1179(6)$ with data shown as a function of the linear lattice size L [73].

A distinct feature of the spectrum is the emergence of a low-lying scalar glueball [73]. The low-lying scalar spectrum remains incomplete without difficult mixing analysis between the scalar fermionic bound state and the scalar glueball. The anomalous dimension $\gamma_{*}$ from conformal scaling, $\mathrm{M} \sim$ const $\cdot \mathrm{m}^{1 / 1+\gamma_{*}}$, is found to be consistent with direct determination from the mode number function as presented earlier [73]. Sampling the expected topological charge distribution in BSM lattice simulations is often slow and presents additional problems to circumvent [77]. Rapid sampling of the topology is reported in the MWT model [73] not effecting the observed spectrum.

Conformal finite size scaling analysis using finite volume data was also performed in the MWT model consistent with other determinations of $\gamma_{*}$ [73]. This method will be described next with application to the model with twelve fermions in the fundamental representation of the SU(3) gauge group, another frequently discussed model in the conformal lattice BSM paradigm.

\subsection{Twelve fermion flavors in the fundamental representation of the $\mathrm{SU}(3)$ gauge group}

This model is very close to the conformal edge, without definitive results inside, or outside of the CW. Its curious and controversial lattice history is difficult and unnecessary to summarize. I will focus on conference contributions and their implications. 


\section{Brief summary of new conference results:}

Itou reported an IRFP in the model from the running coupling of twisted Polyakov loop correlators $[119,120]$. In contrast, Onogi did not reach conclusions using the exact same method in a different data set and different analysis [118]. The Boulder group showed MCRG results which in their interpretation supports the IRFP [67]. New methods, like the gradient flow coupling with high precision data and analysis, are needed to have any chance to settle the fate of the IRFP in the beta function of the model.

The LatKMI group reported consistency with asymptotic conformal scaling $\mathrm{M} \sim$ const $\cdot \mathrm{m}^{1 / 1+\gamma_{*}}$ in their infinite volume extrapolated spectroscopy data [63]. This is not in agreement with some earlier results also based on extrapolated infinite volume data [23] in significantly lower pseudoscalar mass range where conformal scaling should work better. The LatKMI group works at weaker gauge coupling which should help to better control lattice cutoff effects but forces the pseudo-scalar mass range in the $\mathrm{aM}_{\pi}=0.3$ range and higher. Earlier work, using data in [23], invoked additional polynomial terms in the fermion mass $m$ to bring apparent agreement with conformal scaling [27]. I find the results confusing and unsettled. LatKMI results on the scalar mass will be discussed in Section 4.

As a new development, the Boulder group reported results on the anomalous dimension of $\bar{\psi} \psi$ and conformal finite size scaling (FSS) analysis, both consistent with conformality in their interpretation $[68,69]$. Their work, as discussed next, will further illustrate the use of lattice tools in difficult models on the conformal edge.

\section{Conformal finite size scaling:}

The expected leading FSS form for any mass M, or for $\mathrm{F}_{\pi}$, scaled with the linear size $\mathrm{L}$ of the spatial volume, is given by a scaling function $\mathrm{L} \cdot \mathrm{M}=\mathrm{f}(\mathrm{x})$ where $\mathrm{x}=\mathrm{L} \cdot \mathrm{m}^{1 / 1+\gamma}$ is the conformal scaling variable. The scaling form sets in close to the critical surface for small $\mathrm{m}$ values. The scaling functions $\mathrm{f}(\mathrm{x})$ can depend on the quantum numbers of the states but the scaling variable is expected to have the same form with identical $\gamma$ exponent in each quantum number channel [42-45]. It was known before that without subleading conformal scaling correction conformal FSS analysis was not consistent with IRFP when applied to the model $[24,121]$. This is illustrated in Figure 6
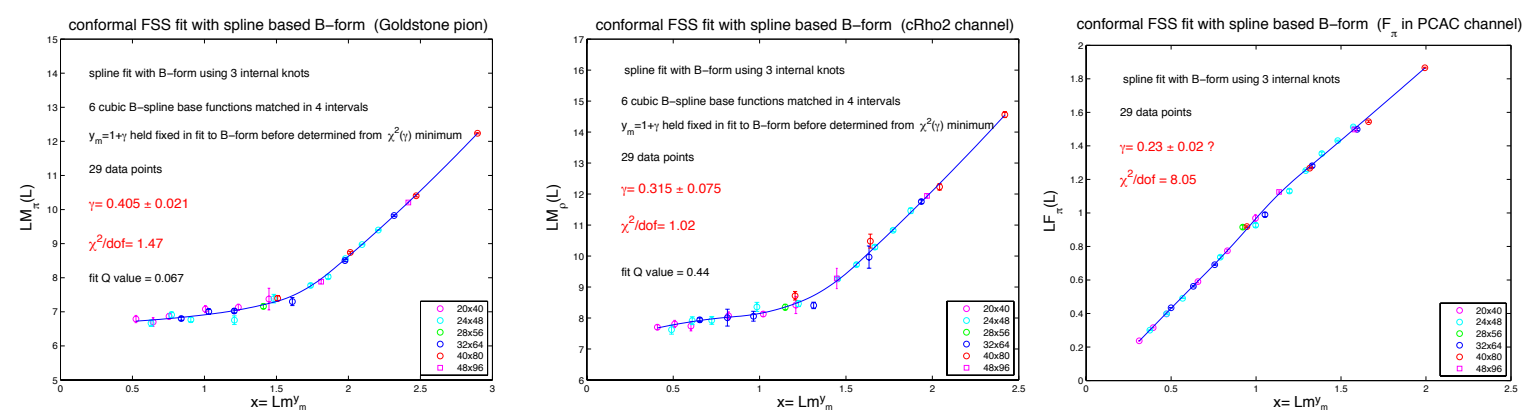

Figure 6: Conformal FSS fits using spline based B-forms in three different channels. The fits are preformed in each channel separately with question mark on $\gamma$ of the right panel indicating difficulties of error estimates in bad fits of $\mathrm{F}_{\pi}[24,121]$.

with a general fitting procedure to the scaling functions using B-forms of spline functions without 
any further restrictions or ad hoc assumptions on the form of the scaling functions [24,121]. The (in)consistency of the $\gamma$ exponents in the pion and rho channels is perhaps acceptable but conformal FSS of the pion deacy constant was seen as complete failure.

It was noted by the same group that in sub-leading order there are conformal FSS scaling violation effects which are exhibited as a combined cutoff and L-dependent leading correction with the modified form $\mathrm{L} \cdot \mathrm{M}=\mathrm{f}(\mathrm{x})+\mathrm{L}^{-\omega} \mathrm{g}(\mathrm{x})$ where the scaling correction exponent $\omega$ is determined at the infrared fixed point (IRFP) $\mathrm{g}_{*}$ of the $\beta$-function as $\omega=\beta^{\prime}\left(\mathrm{g}_{*}\right)[24,121]$. This assumes that the mass deformation away from the critical surface is the only relevant perturbation around the IRFP and $\omega$ is the highest scaling correction exponent. Close enough to the critical surface it dominates any other corrections which are supressed then by higher inverse powers of $\mathrm{L}$. To detect the leading scaling violation effect requires high precision data with fits to independent scaling functions $f(x)$ and $g(x)$ and the critical exponent $\omega$. In the high quality data set of $[24,121]$, designed for conformal FSS, far extending the data set of [23], it was not possible to successfully detect scaling violation effects consistently and explain failure in the $\mathrm{F}_{\pi}$ channel.

In new contribution to the conference, the Boulder group applied the subleading corrections, ignoring the $\mathrm{x}$-dependence of the scaling function $\mathrm{g}(\mathrm{x})$ and reporting good results in support of the IRFP [69]. The results are illustrated in Figure 7 for the pseudo-scalar mass at a selected value of the bare gauge coupling approximately matching the gauge coupling of $[24,121]$. The wide range
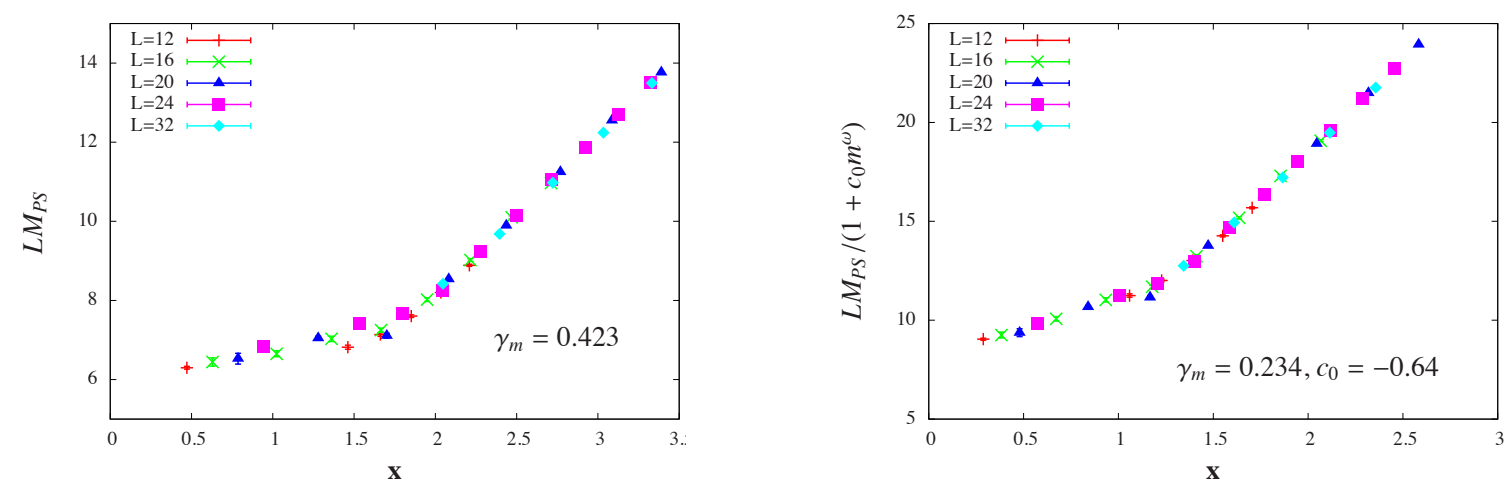

Figure 7: The best curve collapse fits for the pseudo-scalar mass at $\beta_{\mathrm{F}}=4.0$ of the HYP action in [69]. Both panels show $M_{\pi} L$ as the function of the scaling variable $x=m^{1 / y_{m}} L$. Left: Fits considering only the leading relevant operator. Right: Fits taking into account the leading scaling violation correction with further notations in the paper [69].

of gauge couplings is an important development in the conformal FSS Boulder analysis as applied to the model [69]. Without further clarification of the problematic $\chi^{2}$ fit in the $\mathrm{F}_{\pi}$ channel the new analysis perhaps remains incomplete with the original problem persisting in that channel $[24,121]$. Future work would also require to show consistency of the $\omega$ exponent in the beta function and the subleading scaling correction.

\section{Amomalous dimension of the chiral condensate:}

A scale-dependent effective mass anomalous dimension is defined and calculated by the Boulder group using the mode number function [68]. Figure 8 summarizes the new results for the effective anomalous dimension as a function of the scale set by the eigenvalues of the Dirac operator [68]. Gauge couplings are in the wide range $3.0 \leq \beta \leq 6.0$ which is a significant development with results approximately volume independent. The results ar interpreted as follows. All couplings 


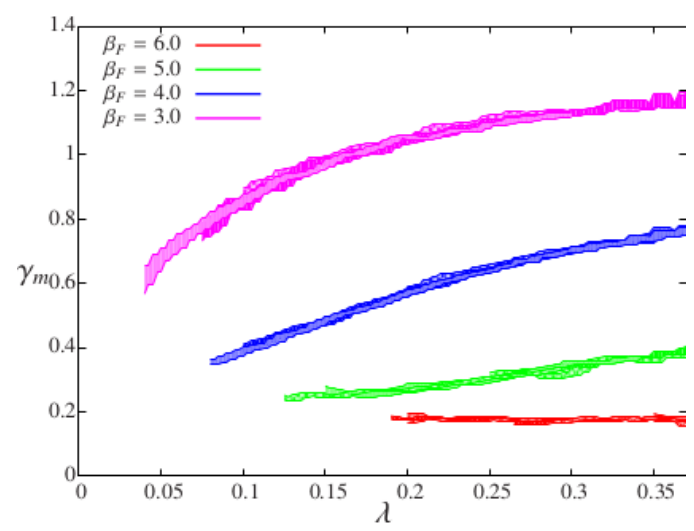

Figure 8: The effective mass anomalous dimension is plotted from stochastic mode number measurements [68]. The largest lattice volume was $48^{4}$ at $\beta=6.0$ and $32^{4}$ at the other gauge couplings [68].

$\beta \leq 5.0$ are outside the weak coupling scaling regime. At the largest value of $\beta$ continuum like scaling behavior sets in, characteristic of sufficiently small lattice spacing. In the infrared limit all data are consistent with the attraction of the conformal IRFP [68]. Independent checks of the interpretation by other groups is a very important next step for continued progress.

\section{Light Higgs near conformality (dilaton-like?)}

The anticipation of light composite scalars near the conformal window can be explained in the following setting. We know that QCD with two massless fermion flavors in the fundamental representation of the $\mathrm{SU}(3)$ color gauge group is very far from the CW and does not exhibit approximate scale invariance in the far infrared. The sigma particle of the model is elusive and heavy on the scale of the pion decay constant $\mathrm{F}_{\pi}$. There is a dramatic change in the position of the model on the navigation map of Figure 1 if the massless fermion doublet is coupled to the SU(3) gauge group in the two-index symmetric representation with six independent color components of the fermions (hence the frequently used name: sextet model) [17,77, 136-139]. Suddenly the model is sitting very close to the conformal edge, scale invariant, or nearly scale invariant in the far infrared. Results supporting near-conformal behavior are presented in Section 4.2. Approximate scale invariance in the near-conformal scenario is far different from naively scaled QCD with exciting new questions about dilaton-like interpretation for a light scalar, if emerging from the theory. Hence the great theoretical interest.

\subsection{The dilaton and PCDC}

The light dilaton is the pseudo-Goldstone particle of spontaneous breaking of scale invariance and has been featured in recent phenomenological discussions for the interpretation of the Higgs discovery [122-125] with a long history in the SCGT paradigm [9, 17, 122, 126-135]. In strongly interacting gauge theories, like the sextet model under active investigation $[138,139]$, a dilatation current $\mathscr{D}^{\mu}=\Theta^{\mu v} x_{v}$ can be defined from the symmetric energy-momentum tensor $\Theta^{\mu v}$. Although the massless theory is scale invariant on the classical level, from the scale anomaly the dilatation 
current has a non-vanishing divergence, $\partial_{\mu} \mathscr{D}^{\mu}=\Theta_{\mu}^{\mu}$. The PCDC relation,

$$
m_{\sigma}^{2} \simeq-\frac{4}{f_{\sigma}^{2}}\left\langle 0\left|\left[\Theta_{\mu}^{\mu}(0)\right]_{N P}\right| 0\right\rangle,
$$

connects the dilaton mass $m_{\sigma}$ and its decay amplitude $f_{\sigma}$ with the non-perturbative gluon condensate in the energy-momentum tensor. For discussion of the PCDC relation constraining the properties of the dilaton, standard arguments, like in $[122,130,133]$ can be followed.

Predictions for $m_{\sigma}$ when close to the conformal window depend on the behavior of $f_{\sigma}$ and the non-perturbative gluon condensate $\left.G_{\mu \nu}^{a} G^{a \mu \nu}\right|_{N P}$ of the energy-momentum tensor $\Theta^{\mu \nu}$. There are two different expectations about the limit of the gluon condensate to $f_{\sigma}$ ratio when the conformal window is approached. In one interpretation, the right-hand side of the PCDC relation is predicted to approach zero in the limit, so that the dilaton mass $\mathrm{m}_{\sigma}^{2} \simeq\left(\mathrm{N}^{\mathrm{c}} \mathrm{f}-\mathrm{Nf}\right) \cdot \Lambda^{2}$ would parametrically vanish when the conformal limit is reached. The $\Lambda$ scale is defined where the running coupling becomes strong to trigger chiral symmetry breaking $(\chi \mathrm{SB})$. The formal parameter $\mathrm{N}^{\mathrm{c}} \mathrm{f}-\mathrm{Nf}$ with the non-physical (fractional) critical number of fermions vanishes when the conformal phase is reached [130]. In an alternate and more widely accepted scenario the right-hand side ratio of the PCDC remains finite in the conformal limit and a residual dilaton mass is expected when scaled with $f_{\sigma} \simeq \Lambda[122,133]$.

Dilaton, or "just Higgs impostor?" :

Composite BSM models close to the $\mathrm{CW}$ will exhibit $\chi \mathrm{SB}$ with a Goldstone pion spectrum. When coupled to the electroweak sector, electroweak symmetry breaking with the Higgs mechanism is realized. The very small beta function (walking) and $\chi \mathrm{SB}$ are not sufficient to guarantee a light dilaton state if scale symmetry breaking and $\chi \mathrm{SB}$ are entangled in a complicated way. It is far from clear that the dilaton mechanism is naturally realized in strong dynamics. However, a light Higgslike scalar is still expected to emerge near the conformal window as a composite state with $0^{++}$ quantum numbers, not necessarily with dilaton interpretation. This scalar state has to be light but not required to exhibit exactly the observed $126 \mathrm{GeV}$ mass. The light scalar from composite strong dynamics is expected to get lighter from electroweak loop corrections, most likely dominated by the large negative mass shift from the top quark loop [136]. In Section 4.2 and Section 4.3 I will discuss three models with light scalars, reported at the conference, as we are searching for candidate Higgs impostors in viable model building.

\subsection{The light scalar of the sextet model}

The sextet representation of the fermion doublet is the important ingredient which brings the model close to the conformal window as suggested by the small beta function of a recent paper [139]. The statistical accuracy of the very small beta function could not resolve the existence of the IRFP from slow walking. However, when combined with the observation of chiral symmetry breaking [138], confirmed at the conference from the chiral limit of small fermion mass deformations [79], the overall consistency requires the model to be close to the conformal window with a very small non-vanishing $\beta$-function (see, also $[49,50,78]$ ).

\section{Candidate Higgs impostor:}

The model exhibits the simplest composite Higgs mechanism with three Goldstone pions of $\chi \mathrm{SB}$ 
transforming into the Electroweak bosons. The light scalar emerged very recently as reported at the conference [77]. This candidate Higgs impostor is identified as the $0^{++}$isospin singlet state in the

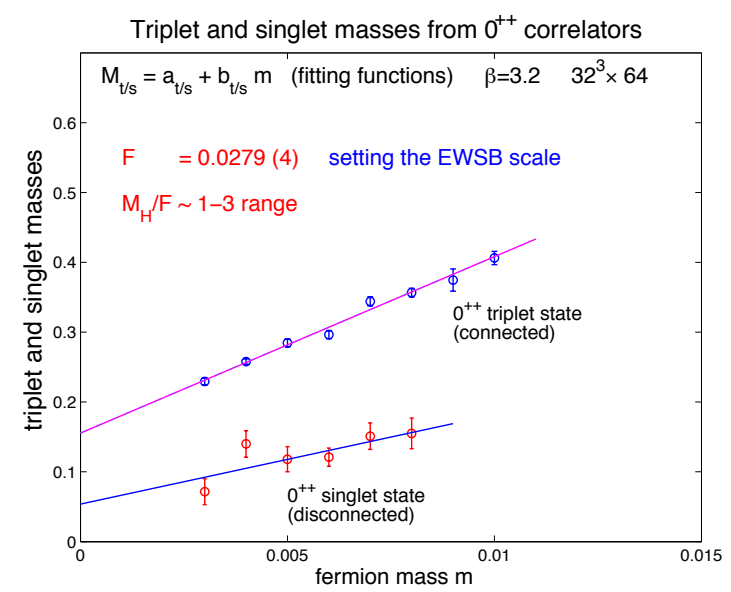

Figure 9: Preliminary results on the emerging light scalar in the sextet model from $32^{3} \times 64$ lattices at gauge coupling $\beta=3.20$. Higher statistics and more comprehensive analysis are required for a more robust extrapolation to the chiral limit [77].

spectrum as shown in Figure 9. The scale $F=(V E V)$, with VEV $=246 \mathrm{GeV}$ from the Electroweak theory, is set in lattice spacing units by the decay constant of the pseudo-scalar pion in the chiral limit. The large split from the isotriplet $0^{++}$state indicates the important role of disconnected vacuum diagrams, as explained in Section 4.3.

\section{Chiral condensate:}

New results were presented at the conference to confirm chiral symmetry breaking in the massless fermion limit of the model [79]. An important consistency check on the fermion mass dependence of the chiral condensate $\bar{\psi} \psi$ and the pion spectrum is the GMOR relation $\langle\bar{\psi} \psi\rangle=M_{\pi}^{2} F_{\pi}^{2} / m$ for two fermion flavors. The Goldstone mass spectrum analysis is shown on the left panel of Figure 10. The variation with mass is less than for the directly-measured condensate, but is still sizable. A quadratic extrapolation to the chiral limit describes the data well, which is consistent with earlier independent fits of $\mathrm{M}_{\pi}^{2}$ and $\mathrm{F}_{\pi}$ in Ref. [138]. However the extrapolated value from the GMOR relation differs from the extrapolations of the directly-measured original and subtracted condensate at gauge coupling $\beta=3.2$ [138]. This discrepancy requires further investigation including cutoff effects from extended data sets of several gauge couplings.

The chiral condensate was independently determined from the eigenvalue density of the Dirac operator for small eigenvalues of gauge configurations as reported at the conference [79]. The eigenvalue density for the largest lattice volume $48^{3} \times 96$ at the lightest fermion mass $\mathrm{m}=0.003$ is shown on the right panel panel of Figure 10. The eigenvalue density increases slowly with $\lambda$ and at this fermion mass the $\rho(\lambda=0)$ density is quite close to the chiral extrapolation of the directlymeasured condensate $\langle\bar{\psi} \psi\rangle$. Topology and the zero modes of the Dirac spectrum from the index theorem should be an important goal of future investigations of the model.

\section{The resonance spectrum:}

It is important to investigate the chiral limit of composite hadron states separated by a gap from the 

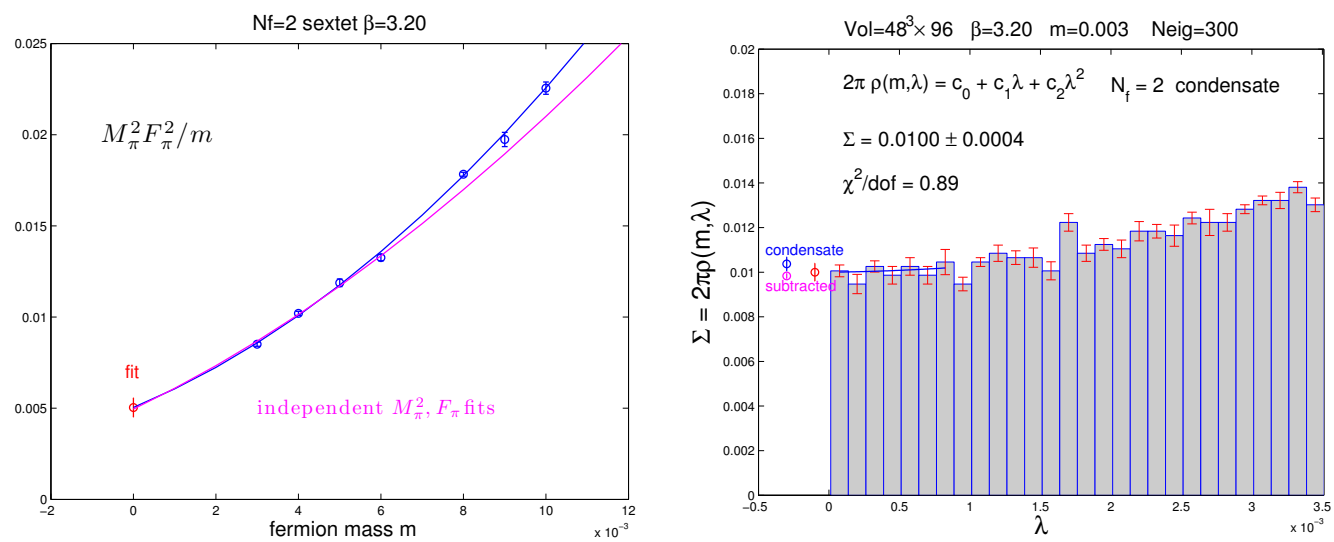

Figure 10: On the left panel the fermion condensate is shown as determined via the GMOR relation. The blue curve is a quadratic fit of all ratio data for $\mathrm{m}=0.003-0.008$, the magenta curve is a combination of separate fits of $\mathrm{M}_{\pi}^{2}$ and $\mathrm{F}_{\pi}$ for $\mathrm{m}=0.003-0.006$. On the right panel the eigenvalue density is shown on $48^{3} \times 96$ lattice volumes of gauge configurations. The extrapolation of the eigenvalue density (red) is compared with the directly measured condensate $\langle\bar{\psi} \psi\rangle$ (blue) and the subtracted condensate (magenta) [79].

Goldstones and the light scalar. Resonance masses of parity partners also provide important information with split parity masses in the chiral limit. This is particularly helpful not only to confirm $\chi \mathrm{SB}$ but to obtain a first estimate on the $\mathrm{S}$ parameter for probing the model against Electroweak precision tests [154]. A remarkable spectrum is emerging within the reach of the LHC14 run as sketched in Figure 11.

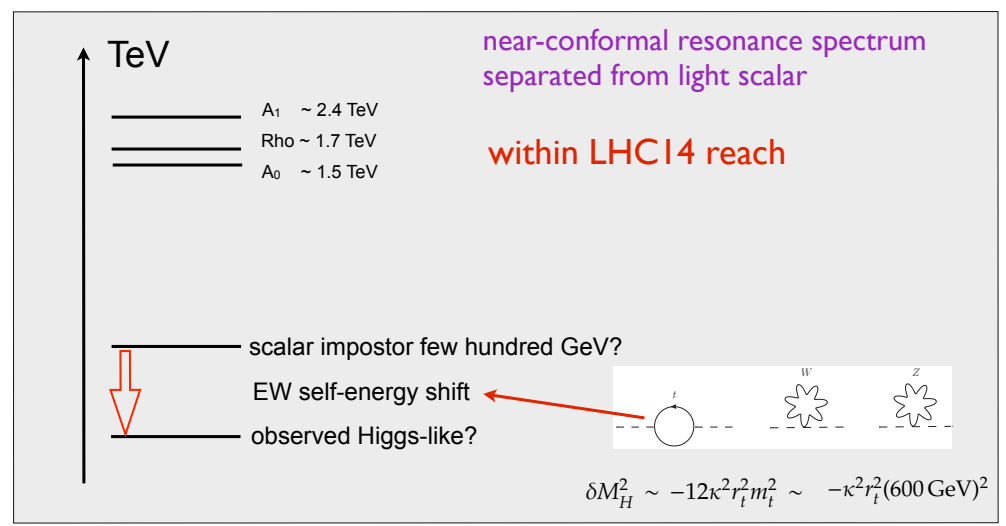

Figure 11: Resonance spectrum based on a single gauge coupling $\beta=3.2$. The control of physical scale setting will require extended data sets of several gauge couplings. Mass shift from Electroweak self-energy diagrams is shown schematically [136].

\subsection{Methodology and scalars in the fundamental fermion representation}

The staggered lattice fermion formulation was used in two independent pilot studies by the LatKMI group and the Lattice Higgs Collaboration $\left(\mathrm{L}_{\mathrm{at}} \mathrm{HC}\right)$ to calculate light scalar states in models with fermions in the fundamental and sextet representations of the SU(3) color gauge group.

\section{$\mathbf{N f}=\mathbf{1 2}$ and $\mathbf{N f}=\mathbf{8}$ :}

The LatKMI group deserves credit to publish first [140] the $\mathrm{Nf}=12$ scalar with further results re- 
ported at the conference $[63,64]$. A distinct additional feature of the LatKMI results is the emergence of a low-lying scalar glueball [64]. Independently, results of the $\mathrm{L}_{\mathrm{at}} \mathrm{HC}$ group were posted in the USQCD BSM white paper submitted to the DOE around the same time [141]. The pilot study of the $\mathrm{L}_{\mathrm{at}} \mathrm{HC}$ group mostly focused on testing the platform of the methodolgy with primary interest in the sextet model [77]. The two groups use similar but not identical technologies with results which match reasonably well, as shown in Figure 12 for the $\mathrm{Nf}=12$ model.
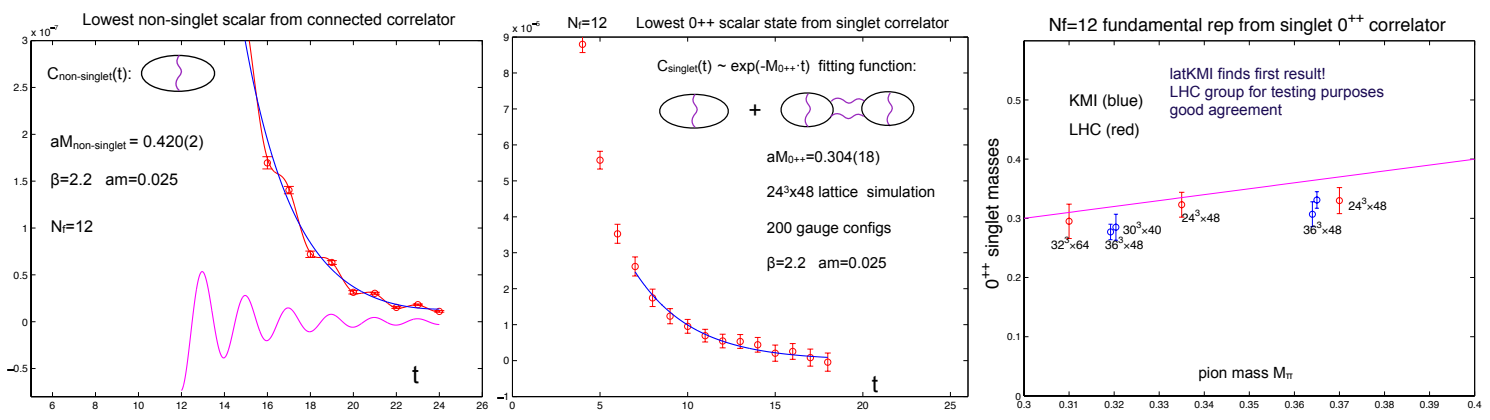

Figure 12: Tests of the light scalar in the model with $\mathrm{Nf}=12$ flavors in the $\mathrm{SU}(3)$ representation from the LatKMI group and the $\mathrm{L}_{\mathrm{at}} \mathrm{HC}$ group. The tree-level Symanzik-improved gauge action with staggered fermions is used and the HMC algorithm is employed [140,141]. On the left panel, at gauge cooupling $\beta=2.20$ and fermion mass am $=0.025$, results from 200 gauge configurations is shown from the $\mathrm{L}_{\mathrm{at}} \mathrm{HC}$ group [141]. A typical non-singlet correlator (red points) has a non-oscillating contribution from the iso-vector $\mathrm{a}_{0}$ scalar (fitted as blue curve) and an oscillating contribution from the parity partner $\pi_{\mathrm{sc}}$ (fitted as magenta curve). The red curve is the combination of the two fits. The panel in the middle shows a typical singlet correlator with non-oscillating contribution from the iso-singlet $\mathrm{f}_{0}$ scalar state (fitted as blue curve). The oscillating contribution $\eta_{\mathrm{SC}}$ is not detectable within statistical fluctuation. On the right panel, matching results are shown from the latKMI collaboration [140] with comparable accuracy to the results of the $\mathrm{L}_{\mathrm{at}} \mathrm{HC}$ group. The magenta curve indicates the $m_{\pi}$ position, for comparison.

A staggered operator which creates a state that lies in the spin-taste representation $\Gamma_{S} \otimes \Gamma_{T}$ also couples to one lying in the $\gamma_{4} \gamma_{5} \Gamma_{S} \otimes \gamma_{4} \gamma_{5} \Gamma_{T}$ representation. Thus a staggered meson correlator has the general form

$$
\mathrm{C}(\mathrm{t})=\sum_{\mathrm{n}}\left[\mathrm{A}_{\mathrm{n}} \mathrm{e}^{-\mathrm{m}_{\mathrm{n}}\left(\Gamma_{\mathrm{S}} \otimes \Gamma_{\mathrm{T}}\right) \mathrm{t}}+(-1)^{\mathrm{t}} \mathrm{B}_{\mathrm{n}} \mathrm{e}^{-\mathrm{m}_{\mathrm{n}}\left(\gamma_{4} \gamma_{5} \Gamma_{\mathrm{S}} \otimes \gamma_{4} \gamma_{5} \Gamma_{\mathrm{T}}\right) \mathrm{t}}\right]
$$

with oscillating contributions from parity partner states. For the scalar meson $\left(\Gamma_{S} \otimes \Gamma_{T}=1 \otimes 1\right)$, the parity partner is $\gamma_{4} \gamma_{5} \otimes \gamma_{4} \gamma_{5}$ which corresponds to one of the pseudo-scalars in the analysis. For

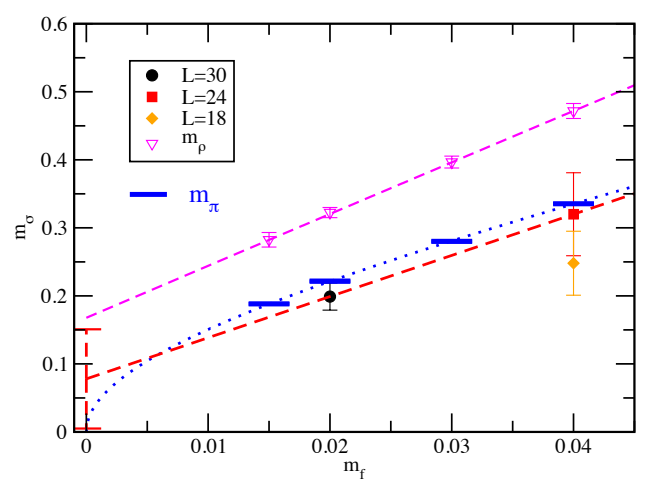

Figure 13: The flavor-singlet scalar mass $m_{\sigma}$ is shown for $\mathrm{Nf}=8$ as a function of $\mathrm{m}_{\mathrm{f}}$ in several lattice volumes [65]. For comparison, the masses $\mathrm{m}_{\pi}$ and $\mathrm{m}_{\rho}$ are also shown from [142].

flavor singlet mesons, the correlator is of the form $\mathrm{C}(\mathrm{t})=\mathrm{C}_{\text {conn }}(\mathrm{t})+\mathrm{C}_{\text {disc }}(\mathrm{t})$ where $\mathrm{C}_{\text {conn }}(\mathrm{t})$ is the 
correlator coupled to the non-singlet meson state and $\mathrm{C}_{\mathrm{disc}}(\mathrm{t})$ is the contribution of disconnected fermion loops in the annihilation diagram. These correlators were used in the $\mathrm{Nf}=12$ analysis as shown in Figure 12. They were also used in the sextet analysis with results preseneted in Section 4.2 and in the $\mathrm{Nf}=8$ analysis as well. LatKMI reported at the conference the first results of a light scalar in the $\mathrm{Nf}=8$ model [65] as shown in Figure 13.

\section{Composite Higgs as a scalar pseudo-Nambu-Goldstone boson}

Non-perturbative results are emerging from this important BSM lattice initiative which I will briefly sketch here. The Lattice Strong Dynamics (LSD) collaboration is actively pursuing the paradigm and the recent USQCD BSM white paper provides more technical details [141].

It is natural to explore strong gauge dynamics where the light composite Higgs is identified with a scalar pseudo-Nambu-Goldstone boson (PNGB) which has zero mass before the Electroweak interactions are turned on. The PNGB Higgs mechanism [143] plays an important role in little Higgs theories [144, 145] and minimal conformal technicolor [146]. In little Higgs theories, an interplay of global symmetries is devised in an effective Lagrangian to cancel the quadratic divergences for the Higgs mass to one loop. This provides a weakly coupled effective theory with little fine tuning up to energies scales in the 5-10 $\mathrm{TeV}$ range.

UV complete strongly coupled lattice gauge theories use fermions in real or pseudo-real representations of the gauge group in this paradigm, necessary for scalar Goldstone particles [147]. In the chiral limit the Higgs scalar is a scalar Goldstone Boson in the massless spectrum that includes a triplet of pseudo-scalars, required to give masses to the $\mathrm{W}$ and $\mathrm{Z}$. The naturally light Higgs mass is induced by small couplings to the Electroweak sector. The main goal of the lattice approach is to demonstrate that viable UV complete theories exist and can replace the weakly coupled elementary Higgs.

\section{Minimal PNGB Model:}

The minimal PNBG Higgs model consists of a $\mathrm{SU}(2)$ color gauge theory with $\mathrm{Nf}=2$ fundamental massless fermions. Additional sterile flavors with $\mathrm{Nf}>2$ can be added [146] to drive the theory close to or into the conformal window. Because of the pseudo-real properties of $\mathrm{SU}(2)$ color group, the conventional $\mathrm{SU}(\mathrm{Nf})_{\mathrm{L}} \times \mathrm{SU}(\mathrm{Nf})_{\mathrm{R}}$ vector-axial symmetry becomes a larger $\mathrm{SU}(2 \mathrm{Nf})$ flavor symmetry combining the $2 \mathrm{Nf}$ left/right 2 -component chiral spinors. Most-attractive-channel arguments suggest that $\mathrm{SU}(2 \mathrm{Nf})$ will break dynamically to $\mathrm{Sp}(2 \mathrm{Nf})$. If explicit masses are given to $\mathrm{Nf}-2$ flavors, the remaining 2 massless flavors yield the $\mathrm{SU}(4) / \mathrm{Sp}(4)$ coset with 5 Goldstone Bosons: the isotriplet pseudo-scalars to give mass to the $\mathrm{W}, \mathrm{Z}$, and two isosinglet scalars. As a first step, lattice calculations for $\mathrm{Nf}=2$ have been performed [147] using Wilson fermions that give non-perturbative support to the breaking pattern, $\mathrm{SU}(4) \rightarrow \mathrm{Sp}(4)$, favored by the most attractive channel argument in the chiral limit.

To include the effect of the top quark into the lattice simulations, one must add the four Fermi term induced by the top quark loop. The four Fermi term is then recast as a quadratic term coupled to a Gaussian auxiliary field. It is an open question whether or not the four-fermion operator requires an explicit cut-off or whether it becomes a relevant operator due to a large anomalous dimension. Important problems have to be sorted out in the fermion determiant including its sign and the Pfaffian form. When both the diquark and bilinear for the four Fermi term are included 
together, the measure is a Pfaffian, or the square root of the fermionic determinant. An example has been considered recently for staggered fermions [93,148]. This will require continued further study, like the ongoing effort of the LSD collaboration [141].

\section{SUSY on the Lattice}

One natural solution to the problems with the Standard Model Higgs is to incorporate supersymmetry. The Higgs becomes naturally light since it is paired with a fermonic superpartner whose mass is protected by chiral symmetries. There were important SUSY contributions at the conference [81-84] which I cannot discuss individually in the alloted time and space. In the general context, I will sketch some recent lattice SUSY developements with reference for more details in [141].

\section{$\mathcal{N}=1$ super Yang-Mills:}

Credible studies of SUSY theories on the lattice emerged from successfully using domain wall fermions [149] in $\mathscr{N}=1$ super Yang-Mills investigations. Using these ideas one can also plan now lattice studies of super QCD theories. The work in super QCD is important for gaining a detailed understanding of the dynamical breaking of supersymmetry. This has important phenonenological implications since non-perturbative supersymmetry breaking in some high scale hidden sector feeds down to determine the structure of soft breaking terms in any low scale supersymmetric theory, like MSSM. The values of soft parameters are determined by non-perturbative quantities in the high scale theory. An ab initio lattice calculation of such quantities in the high scale theory can thus provide constraints on models of BSM physics. While super QCD is known to have no exact SUSY breaking vacua it does possess many metastable vacua whose lifetimes can be sufficiently large that they can play this role [150]. Thus a detailed understanding of the vacuum structure and strong coupling dynamics of super QCD can strongly constrain possible supersymmetric models. Preliminary work has already demonstrated non-zero gluino condensate in the $\mathrm{SU}(2)$ theory in agreement with theoretical expectations $[149,151]$. A plot of the gaugino condensate vs. residual mass is shown in Figure 14. Building a realistic theory will require to add Nf quarks and their scalar superpartners (squarks), and additionally extend the gauge group to a larger number of colors $\mathrm{N}_{\mathrm{c}}$. To restore SUSY in the continuum limit now will require DWF and tuning of parameters in the squark sector [152].

\section{$\mathcal{N}=\mathbf{4}$ super Yang-Mills:}

As we discussed earlier, there has been renewed interest in the possibility that the Higgs is a pseudodilaton state associated with spontaneous breaking of scale invariance. This is particularly simple to realize in a supersymmetric theory with flat directions, with the prime example of $\mathscr{N}=4$ super Yang-Mills. The dilaton corresponds to translations along such a flat direction. A related recent development has been the construction of lattice theories with some exact supersymmetry at non zero lattice spacing, like $\mathscr{N}=4$ super Yang-Mills. The exact supersymmetry present in the $\mathscr{N}=4$ lattice theory avoids fine tuning problems and deploys lattice simulations to study the non-perturbative structure of this theory [153]. The $\mathscr{N}=4$ lattice action has flat directions which are stable against quantum corrections due to the exact lattice supersymmetry. To realize the spontaneously broken state and the corresponding dilaton in the lattice theory then requires that a non-zero vacuum expec- 


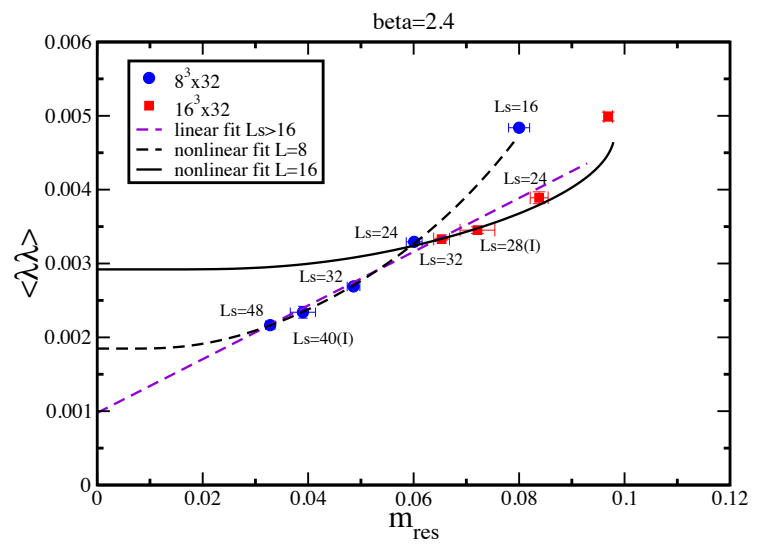

Figure 14: Gaugino condensate vs residual mass for $\mathrm{SU}(2) \mathscr{N}=1$ super Yang-Mills regulated using a domain wall fermion action. The gauge coupling $\beta=2.4$ while the bare fermion mass is set to zero. The data points correspond to different lattice volumes and $\mathrm{L}_{\mathrm{s}}[149,151]$.

tation value of one or more scalar fields kept fixed and the lattice Monte Carlo procedure modifed so that no integration over this mode is carried out.

The conference contributions [81-84] and the two examples I briefly described clearly show that lattice SUSY is actively contributing to the BSM paradigm.

\section{Phenomenological applications of the BSM lattice toolset}

Phenomenological applications are beginning to emerge from lattice investigations. The LSD collaboration plays a very important leading role in these investigations with impressive results reviewed in the USQCD BSM white paper [141].

\subsection{S-parameter}

The $S$ parameter was introduced to analyze oblique Electroweak corrections in precision tests [154]. The experimental value of $S$ depends on the Higgs mass $M_{H}$; with $M_{H}=126 \mathrm{GeV}$ the constraint is $\mathrm{S}=0.03 \pm 0.10$, consistent with the standard model [155]. QCD-like new strong dynamics would predict $\mathrm{S} \gtrsim 0.3$; this was originally obtained by scaling up experimental QCD data for vector (V) and axial-vector (A) spectral functions to the electroweak scale [154], and has been confirmed by lattice QCD calculations [156-158].

To see how non-QCD-like dynamics may change the situation, consider

$$
\mathrm{S}=4 \pi \mathrm{N}_{\mathrm{D}} \lim _{\mathrm{Q}^{2} \rightarrow 0} \frac{\mathrm{d}}{\mathrm{dQ}^{2}} \Pi_{\mathrm{V}-\mathrm{A}}\left(\mathrm{Q}^{2}\right)-\Delta \mathrm{S}_{\mathrm{SM}}, \quad 4 \pi \Pi_{\mathrm{V}-\mathrm{A}}^{\prime}(0)=\frac{1}{3 \pi} \int_{0}^{\infty} \frac{\mathrm{ds}}{\mathrm{s}}\left[\mathrm{R}_{\mathrm{V}}(\mathrm{s})-\mathrm{R}_{\mathrm{A}}(\mathrm{s})\right],
$$

where $\Pi_{\mathrm{V}-\mathrm{A}}\left(\mathrm{Q}^{2}\right)$ is related to a dispersive integral of spectral functions. The near restoration of chiral symmetry with approximate parity doubling will lead to strong cancellations between $R_{V}(s)$ and $\mathrm{R}_{\mathrm{A}}(\mathrm{s})$ and therefore reduce $\mathrm{S}$ as expected near the conformal window.

On the lattice, $\Pi_{\mathrm{V}-\mathrm{A}}\left(\mathrm{Q}^{2}\right)$ is measured from $\mathrm{V}$ and $\mathrm{A}$ two-point current correlation functions. Existing calculations use overlap or domain wall fermions, for which good chiral and flavor symmetries lead to large cancellations of lattice artifacts in the $\mathrm{V}-\mathrm{A}$ difference, and to equal renormalization factors $\mathrm{Z}_{\mathrm{V}}=\mathrm{Z}_{\mathrm{A}}[156,157,159]$. These formulations also allow direct connection to contin- 


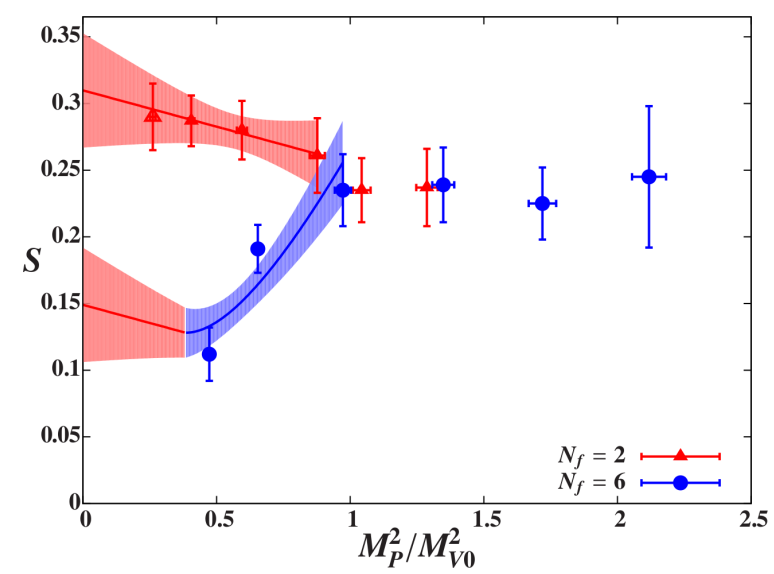

Figure 15: The $\mathrm{S}$ parameter for $\mathrm{SU}(3)$ gauge theories with $\mathrm{Nf}=2$ and 6 fundamental fermions, from Ref. [159]. $\mathrm{M}_{\mathrm{P}}$ is the pseudo-scalar mass, while $\mathrm{M}_{\mathrm{V} 0}$ is the vector meson mass in the chiral limit. The $\mathrm{Nf}=6$ theory has 35 pseudo-NGBs, and here it is assumed that 32 of them have mass $\approx 0.6 \mathrm{M}_{\mathrm{V} 0}$.

uum chiral perturbation theory, where the $\mathrm{S}$ parameter corresponds to the low-energy constant $\mathrm{L}_{10}$. So far few lattice studies of S have been completed: Refs. [156-158] investigated QCD-like technicolor, and relative to those results Ref. [158] observed a significant reduction in $\mathrm{S}$ with $\mathrm{Nf}=6$ light fermions (Figure 15). Ref. [160] reported some qualitative investigations of $\Pi_{V-A}\left(Q^{2}\right)$ for fermions in the sextet representation of $\mathrm{SU}(3)$.

\subsection{WW Scattering on the Lattice}

The scattering of $\mathrm{W}$ and $\mathrm{Z}$ gauge bosons is sensitive to deviations from the Standard Model in the electroweak symmetry-breaking sector. In W/Z scattering at low energies one "integrates out"

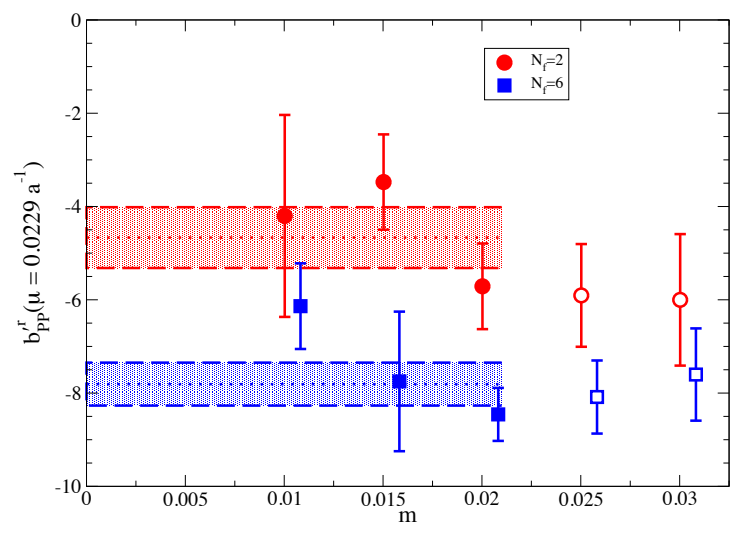

Figure 16: From [161], comparison of chiral parameter $b_{P P}^{\prime r}$ obtained from maximal-isospin $\pi-\pi$ scattering in SU(3) gauge theories with $\mathrm{Nf}=2$ (red) and $\mathrm{Nf}=6$ (blue) light fermion species. The parameter $\mathrm{b}_{\mathrm{PP}}^{\mathrm{r}}$ is sensitive to a combination of low-energy constants which would be relevant for WW scattering, hinting that the scattering rate may be enhanced in theories of electroweak symmetry breaking constructed with larger Nf.

the heavier states to work in an effective field theory resembling the Standard Model [162-164]. The first deviations would appear in two-, three-, and four-point functions of the electroweak gauge 
bosons, corresponding to oblique corrections [154,158], anomalous triple-boson vertices, and W/Z boson scattering.

A recent lattice study has focused on the low-energy constants relevant for WW scattering in $\mathrm{SU}(3)$ gauge theories with $\mathrm{Nf}=2$ and 6 light fermion species [161]. In the strongly-coupled gauge theory, the relevant physical process to determine these low-energy constants is $\pi$ - $\pi$ scattering (these "pions" will become the longitudinal modes of the $\mathrm{W}$ bosons in a composite Higgs theory.) In the initial work, only the extraction of a combination of the relevant low-energy constants was possible; calculation of scattering lengths in other channels and the use of partially-quenched fermion masses may be necessary to separate the individual constants. An interesting trend was observed in the initial study, with the measured combination of low-energy constants showing a significant enhancement from $\mathrm{Nf}=2$ to $\mathrm{Nf}=6$ (see Figure 16). If this trend continues, theories with greater fermion content may give rise to greatly enhanced WW scattering rates, which could be observed at the LHC.

\subsection{Composite Dark Matter}

Composite dark matter models can have unique phenomenological signatures. In these models, an electroweak-neutral composite dark matter candidate can be formed as a bound state of electroweak-charged fundamental particles, bound together by a new strong gauge force. The charged constituents give rise to the necessary interactions in the early universe, but the interactions of the neutral state with ordinary matter in the present Universe will be greatly suppressed. In scenarios of dynamical electroweak symmetry breaking, in which the Higgs boson itself is a composite state, the presence of additional bound states which can play the role of dark matter is natural and the couplings to the electroweak sector are particularly well-motivated $[165,167,168]$.

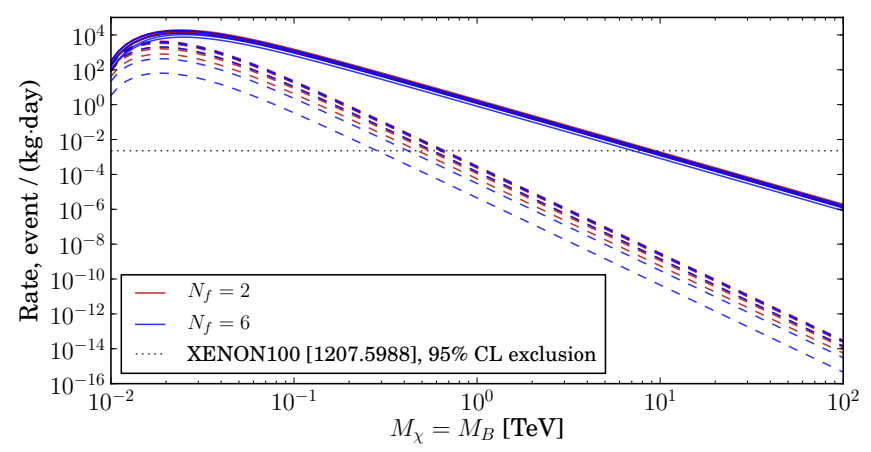

Figure 17: From [104], calculated Xenon 100 event rates based on simulation results for the charge radius (dashed) and magnetic moment (solid) of baryonic dark matter in three-color gauge theories with $\mathrm{Nf}=2$ and $\mathrm{Nf}=6$ light fermion species. Bounds arising from the magnetic moment interaction are quite strong, of order $10 \mathrm{TeV}$ based on the latest Xenon100 data [169].

In the context of composite dark matter, there are many observables of interest, but one set of observables which are directly relevant to experiment are the electromagnetic form factors, which determine the electroweak interaction strength of dark matter with ordinary matter. Recently, a lattice calculation of the magnetic moment and charge radius has been carried out in gauge theories with $\mathrm{Nf}=2$ and $\mathrm{Nf}=6$ light fermion species in the $\mathrm{SU}(3)$ representation of the gauge group. $\mathrm{Nf}=2$ corresponds to $\mathrm{QCD}$, while the presence of extra fermions in the $\mathrm{Nf}=6$ theory change the 
dynamical properties in comparison with QCD [28]. Results for the charge radius and magnetic moment, converted to direct-detection constraints for the recent Xenon100 experimental release, allow the exclusion of composite dark matter based on these models for masses up to $10 \mathrm{TeV}$ as shown in Figure 17 [104].

\section{Acknowledgments}

The author would like to thank the organizers for their kind hospitality at the most enjoyable Lattice 2013 conference. This work was supported by the DOE under grant DE-SC0009919.

\section{References}

[1] S. Chatrchyan et al. [CMS Collaboration], Phys. Lett. B 716, 30 (2012) [arXiv:1207.7235].

[2] G. Aad et al. [ATLAS Collaboration], Phys. Lett. B 716, 1 (2012) [arXiv:1207.7214].

[3] S. Weinberg, Phys. Rev. D 19, 1277 (1979).

[4] L. Susskind, Phys. Rev. D 20, 2619 (1979).

[5] S. Dimopoulos, L. Susskind, Nucl. Phys. B155, 237-252 (1979).

[6] E. Eichten, K. D. Lane, Phys. Lett. B90, 125-130 (1980).

[7] E. Farhi and L. Susskind, Phys. Rept. 74, 277 (1981).

[8] B. Holdom, Phys. Lett. B150, 301 (1985).

[9] K. Yamawaki, M. Bando, K. -i. Matumoto, Phys. Rev. Lett. 56, 1335 (1986); Phys. Lett. B 178, 308 (1986).

[10] T. Appelquist, L. C. R. Wijewardhana, Phys. Rev. D36, 568 (1987).

[11] W. E. Caswell, Phys. Rev. Lett. 33, 244 (1974).

[12] T. Banks and A. Zaks, Nucl. Phys. B 196, 189 (1982).

[13] W. J. Marciano, Phys. Rev. D 21, 2425 (1980).

[14] J. B. Kogut, J. Shigemitsu and D. K. Sinclair, Phys. Lett. B 145, 239 (1984).

[15] T. Appelquist, M. Piai, R. Shrock, Phys. Rev. D69, 015002 (2004).

[16] F. Sannino, K. Tuominen, Phys. Rev. D71, 051901 (2005).

[17] D. D. Dietrich, F. Sannino, K. Tuominen, Phys. Rev. D72, 055001 (2005).

[18] M. A. Luty, T. Okui, JHEP 0609, 070 (2006).

[19] D. D. Dietrich, F. Sannino, Phys. Rev. D75, 085018 (2007).

[20] M. Kurachi, R. Shrock, JHEP 0612, 034 (2006).

[21] Z. Fodor, K. Holland, J. Kuti, D. Nogradi and C. Schroeder, Phys. Lett. B 681, 353 (2009).

[22] Z. Fodor, K. Holland, J. Kuti, D. Nogradi and C. Schroeder, PoS LAT2010, 060 (2010) [arXiv:1103.5998 [hep-lat].

[23] Z. Fodor, K. Holland, J. Kuti, D. Nogradi, C. Schroeder, Phys. Lett. B 703, 348 (2011).

[24] Z. Fodor, K. Holland, J. Kuti, D. Nogradi, C. Schroeder and C. H. Wong, PoS LAT2011, 073 (2011) arXiv:1205.1878 [hep-lat].

[25] T. Appelquist, G. T. Fleming and E. T. Neil, Phys. Rev. Lett. 100, 171607 (2008).

[26] T. Appelquist, G. T. Fleming and E. T. Neil, Phys. Rev. D 79, 076010 (2009),

[27] T. Appelquist, G. T. Fleming, M. F. Lin, E. T. Neil and D. A. Schaich, Phys. Rev. D 84, 054501 (2011) [arXiv:1106.2148 [hep-lat]].

[28] T. Appelquist et al., Phys. Rev. Lett. 104, 071601 (2010).

[29] A. Deuzeman, M. P. Lombardo, E. Pallante, Phys. Lett. B670, 41-48 (2008).

[30] A. Deuzeman, M. P. Lombardo, E. Pallante, Phys. Rev. D82, 074503 (2010). 
[31] A. Deuzeman, M. P. Lombardo, T. N. da Silva and E. Pallante, arXiv:1111.2590 [hep-lat].

[32] A. Hasenfratz, Phys. Rev. D80, 034505 (2009).

[33] A. Hasenfratz, Phys. Rev. D82, 014506 (2010).

[34] A. Cheng, A. Hasenfratz and D. Schaich, arXiv:1111.2317 [hep-lat].

[35] X. -Y. Jin, R. D. Mawhinney, PoS LAT2009, 049 (2009).

[36] X. -Y. Jin, R. D. Mawhinney, PoS LATTICE2010, 055 (2010).

[37] Y. Aoki, T. Aoyama, M. Kurachi, T. Maskawa, K. -i. Nagai, H. Ohki, A. Shibata, K. Yamawaki, and T. Yamazaki, arXiv:1202.4916 [hep-lat].

[38] S. Catterall, F. Sannino, Phys. Rev. D76, 034504 (2007).

[39] S. Catterall, J. Giedt, F. Sannino, J. Schneible, JHEP 0811, 009 (2008).

[40] A. J. Hietanen, J. Rantaharju, K. Rummukainen, K. Tuominen, JHEP 0905, 025 (2009).

[41] A. J. Hietanen, K. Rummukainen, K. Tuominen, Phys. Rev. D80, 094504 (2009).

[42] L. Del Debbio, B. Lucini, A. Patella, C. Pica, A. Rago, Phys. Rev. D82, 014510 (2010).

[43] F. Bursa, L. Del Debbio, L. Keegan, C. Pica, T. Pickup, Phys. Lett. B696, 374-379 (2011).

[44] L. Del Debbio, R. Zwicky, Phys. Rev. D82, 014502 (2010).

[45] L. Del Debbio, B. Lucini, A. Patella, C. Pica and A. Rago, arXiv:1111.4672 [hep-lat].

[46] Y. Shamir, B. Svetitsky, T. DeGrand, Phys. Rev. D78, 031502 (2008).

[47] T. DeGrand, Y. Shamir, B. Svetitsky, Phys. Rev. D82, 054503 (2010).

[48] T. DeGrand, Phys. Rev. D 84, 116901 (2011) [arXiv:1109.1237 [hep-lat]].

[49] J. B. Kogut, D. K. Sinclair, Phys. Rev. D81, 114507 (2010).

[50] J. B. Kogut and D. K. Sinclair, Phys. Rev. D 84, 074504 (2011) [arXiv:1105.3749 [hep-lat]].

[51] E. Bilgici, A. Flachi, E. Itou, M. Kurachi, C. -J D. Lin, H. Matsufuru, H. Ohki, T. Onogi, Phys. Rev. D80, 034507 (2009)

[52] E. Itou, T. Aoyama, M. Kurachi, C. -J. D. Lin, H. Matsufuru, H. Ohki, T. Onogi, E. Shintani, PoS LATTICE2010, 054 (2010).

[53] N. Yamada, M. Hayakawa, K. -I. Ishikawa, Y. Osaki, S. Takeda, S. Uno, PoS LAT2009, 066 (2009).

[54] M. Hayakawa, K. -I. Ishikawa, Y. Osaki, S. Takeda, S. Uno, N. Yamada, [arXiv:1011.2577 [hep-lat]].

[55] R. V. Gavai, Nucl. Phys. B269, 530 (1986).

[56] N. Attig, B. Petersson, M. Wolff, Phys. Lett. B190, 143 (1987).

[57] S. Meyer, B. Pendleton, Phys. Lett. B241, 397-402 (1990).

[58] P. H. Damgaard, U. M. Heller, A. Krasnitz, P. Olesen, Phys. Lett. B400, 169-175 (1997).

[59] S. -y. Kim, S. Ohta, Phys. Rev. D46, 3607-3617 (1992).

[60] F. R. Brown, H. Chen, N. H. Christ, Z. Dong, R. D. Mawhinney, W. Schaffer, A. Vaccarino, Phys. Rev. D46, 5655-5670 (1992).

[61] Y. Iwasaki, K. Kanaya, S. Kaya, S. Sakai, T. Yoshie, Phys. Rev. D69, 014507 (2004).

[62] Y. Iwasaki, arXiv:1212.4343 [hep-lat].

[63] Y. Aoki et al. [ for the LatKMI Collaboration], arXiv:1311.6885 [hep-lat].

[64] Y. Aoki, T. Aoyama, M. Kurachi, T. Maskawa, K. Miura, K. -i. Nagai, H. Ohki and E. Rinaldi et al., arXiv:1310.0963 [hep-lat].

[65] Y. Aoki, T. Aoyama, M. Kurachi, T. Maskawa, K. Miura, K. -i. Nagai, H. Ohki and E. Rinaldi et al., arXiv:1309.0711 [hep-lat].

[66] D. Schaich [USBSM Collaboration], [arXiv:1310.7006 [hep-lat]].

[67] G. Petropoulos, A. Cheng, A. Hasenfratz and D. Schaich, arXiv:1311.2679 [hep-lat].

[68] A. Cheng, A. Hasenfratz, G. Petropoulos and D. Schaich, arXiv:1311.1287 [hep-lat].

[69] A. Hasenfratz, A. Cheng, G. Petropoulos and D. Schaich, arXiv:1310.1124 [hep-lat]. 
[70] Z. Gelzer, Y. Liu, Y. Meurice and D. K. Sinclair, arXiv:1312.3906 [hep-lat].

[71] M. Tomii, M. Hayakawa, K. -I. Ishikawa, S. Takeda and N. Yamada, arXiv:1311.0099 [hep-lat].

[72] T. Appelquist, R. C. Brower, M. I. Buchoff, M. Cheng, G. T. Fleming, J. Kiskis, M. F. Lin and E. T. Neil et al., arXiv:1311.4889 [hep-ph].

[73] L. Del Debbio, B. Lucini, C. Pica, A. Patella, A. Rago and S. Roman, arXiv:1311.5597 [hep-lat].

[74] J. Rantaharju, arXiv:1311.3719 [hep-lat].

[75] A. Athenodorou, E. Bennett, G. Bergner, B. Lucini and A. Patella, PoS LATTICE 2013, 066 (2013) [arXiv:1311.4155 [hep-lat]].

[76] T. DeGrand, Y. Shamir and B. Svetitsky, arXiv:1310.2128 [hep-lat].

[77] Z. Fodor, K. Holland, J. Kuti, D. Nogradi and C. H. Wong, arXiv:1401.2176 [hep-lat].

[78] D. K. Sinclair and J. B. Kogut, arXiv:1311.5208 [hep-lat].

[79] Z. Fodor, K. Holland, J. Kuti, D. Nogradi and C. H. Wong, arXiv:1402.6029 [hep-lat].

[80] A. Hietanen, C. Pica, F. Sannino and U. Sondergaard, arXiv:1311.3841 [hep-lat].

[81] S. Musberg, G. Münster and S. Piemonte, PoS LATTICE 2013, 065 (2013) [arXiv:1311.6312 [hep-lat]].

[82] G. Bergner, I. Montvay, G. Münster, D. Sandbrink and U. D. Özugurel, PoS LATTICE 2013, 483 (2013) [arXiv:1311.1681 [hep-lat]].

[83] K. Steinhauer and U. Wenger, PoS LATTICE 2013, 092 (2013) [arXiv:1311.5403 [hep-lat]].

[84] D. J. Weir, S. Catterall and D. Mehta, arXiv:1311.3676 [hep-lat].

[85] F. Knechtli, K. Yoneyama, P. Dziennik and N. Irges, arXiv:1402.3491 [hep-lat].

[86] L. Del Debbio, R. D. Kenway, E. Lambrou and E. Rinaldi, arXiv:1309.6249 [hep-lat].

[87] K. Kashiwa, H. Kouno, T. Makiyama, T. Misumi, T. Sasaki and M. Yahiro, arXiv:1311.4918 [hep-ph].

[88] G. Cossu, E. Itou, H. Hatanaka, Y. Hosotani and J. -I. Noaki, arXiv:1311.0079 [hep-lat].

[89] A. Maas and T. Mufti, arXiv:1310.7832 [hep-lat].

[90] P. Hegde, G. W. -S. Hou, K. Jansen, B. Knippschild, C. -J. D. Lin, K. -I. Nagai, A. Nagy and K. Ogawa, arXiv:1310.5922 [hep-lat].

[91] P. Hegde, K. Jansen, C. -J. D. Lin and A. Nagy, arXiv:1310.6260 [hep-lat].

[92] M. Wurtz and R. Lewis, arXiv:1310.1121 [hep-lat].

[93] S. Catterall and A. Veernala, arXiv:1311.4417 [hep-lat].

[94] L. Del Debbio, L. Keegan and C. Pena, arXiv:1311.4458 [hep-lat].

[95] R. C. Brower, G. T. Fleming and H. Neuberger, Phys. Lett. B 721, 299 (2013) [arXiv:1212.6190 [hep-lat]].

[96] E. T. Tomboulis, arXiv:1311.5289 [hep-lat].

[97] R. Lohmayer and R. Narayanan, arXiv:1311.2760 [hep-lat].

[98] A. Gonzalez-Arroyo and M. Okawa, arXiv:1311.3778 [hep-lat].

[99] M. G. Perez, A. Gonzalez-Arroyo, L. Keegan and M. Okawa, arXiv:1311.2395 [hep-lat].

[100] G. S. Bali, L. Castagnini, B. Lucini and M. Panero, arXiv:1311.7559 [hep-lat].

[101] J. Ambjorn, A. Görlich, J. Jurkiewicz and R. Loll, Int. J. Mod. Phys. D 22, 1330019 (2013).

[102] M. A. Zubkov, arXiv:1308.3406 [hep-lat].

[103] T. Rindlisbacher and P. de Forcrand, arXiv:1311.4712 [hep-lat].

[104] T. Appelquist et al. [Lattice Strong Dynamics (LSD) Collaboration], Phys. Rev. D 88, no. 1, 014502 (2013) [arXiv:1301.1693 [hep-ph]].

[105] M. Laine, G. Nardini and K. Rummukainen, arXiv:1311.4424 [hep-lat].

[106] Z. Fodor, K. Holland, J. Kuti, D. Nogradi and C. H. Wong, PoS LATTICE 2012, 050 (2012) [arXiv:1211.3247 [hep-lat]].

[107] Z. Fodor, K. Holland, J. Kuti, D. Nogradi and C. H. Wong, JHEP 1211, 007 (2012) [arXiv:1208.1051 [hep-lat]]. [108] M. Lüscher, arXiv:1308.5598 [hep-lat]. 
[109] M. Luscher, R. Narayanan, P. Weisz and U. Wolff, Nucl. Phys. B 384, 168 (1992) [hep-lat/9207009].

[110] P. Fritzsch and A. Ramos, JHEP 1310, 008 (2013) [arXiv:1301.4388 [hep-lat]].

[111] P. Fritzsch and A. Ramos, arXiv:1308.4559 [hep-lat].

[112] P. Fritzsch, A. Ramos and F. Stollenwerk, arXiv:1311.7304 [hep-lat].

[113] J. Rantaharju, K. Rummukainen and K. Tuominen, arXiv:1301.2373 [hep-lat].

[114] L. Del Debbio, L. Giusti, M. Luscher, R. Petronzio and N. Tantalo, JHEP 0602, 011 (2006) [hep-lat/0512021].

[115] L. Giusti and M. Luscher, JHEP 0903, 013 (2009) [arXiv:0812.3638 [hep-lat]].

[116] A. Patella, Phys. Rev. D 86, 025006 (2012) [arXiv:1204.4432 [hep-lat]].

[117] A. Cheng, A. Hasenfratz, G. Petropoulos and D. Schaich, JHEP 1307, 061 (2013) [arXiv:1301.1355 [hep-lat]].

[118] C. -J. D. Lin, K. Ogawa, H. Ohki and E. Shintani, JHEP 1208, 096 (2012) [arXiv:1205.6076 [hep-lat]].

[119] E. Itou, arXiv:1311.2998 [hep-lat].

[120] E. Itou, arXiv:1311.2676 [hep-lat].

[121] Z. Fodor, K. Holland, J. Kuti, D. Nogradi, C. Schroeder and C. H. Wong, PoS LATTICE 2012, 279 (2012) [arXiv:1211.4238 [hep-lat]].

[122] S. Matsuzaki and K. Yamawaki, Phys. Rev. D 86, 035025 (2012) [arXiv:1206.6703 [hep-ph]].

[123] J. Ellis and T. You, arXiv:1207.1693 [hep-ph].

[124] I. Low, J. Lykken and G. Shaughnessy, arXiv:1207.1093 [hep-ph].

[125] D. Elander and M. Piai, arXiv:1208.0546 [hep-ph].

[126] W. A. Bardeen, C. N. Leung and S. T. Love, Phys. Rev. Lett. 56, 1230 (1986).

[127] B. Holdom and J. Terning, Phys. Lett. B 187, 357 (1987); Phys. Lett. B 200, 338 (1988).

[128] V. A. Miransky and K. Yamawaki, Phys. Rev. D 55, 5051 (1997).

[129] W. D. Goldberger, B. Grinstein and W. Skiba, Phys. Rev. Lett. 100, 111802 (2008).

[130] T. Appelquist and Y. Bai, Phys. Rev. D 82, 071701 (2010).

[131] B. Grinstein and P. Uttayarat, JHEP 1107, 038 (2011).

[132] O. Antipin, M. Mojaza and F. Sannino, Phys. Lett. B 712, 119 (2012).

[133] M. Hashimoto and K. Yamawaki, Phys. Rev. D 83, 015008 (2011).

[134] S. Matsuzaki and K. Yamawaki, Phys. Rev. D 86, 115004 (2012) [arXiv:1209.2017 [hep-ph]].

[135] O. Antipin, S. Di Chiara, M. Mojaza, E. Molgaard and F. Sannino, arXiv:1205.6157 [hep-ph].

[136] R. Foadi, M. T. Frandsen and F. Sannino, Phys. Rev. D 87, 095001 (2013) [arXiv:1211.1083 [hep-ph]].

[137] D. K. Hong, S. D. H. Hsu and F. Sannino, Phys. Lett. B 597, 89 (2004) [hep-ph/0406200].

[138] Z. Fodor, K. Holland, J. Kuti, D. Nogradi, C. Schroeder and C. H. Wong, Phys. Lett. B 718, 657 (2012) [arXiv:1209.0391 [hep-lat]].

[139] T. DeGrand, Y. Shamir and B. Svetitsky, Phys. Rev. D 87, 074507 (2013) [arXiv:1201.0935 [hep-lat]].

[140] Y. Aoki, T. Aoyama, M. Kurachi, T. Maskawa, K. -i. Nagai, H. Ohki, E. Rinaldi and A. Shibata et al., Phys. Rev. Lett. 111, 162001 (2013) [arXiv:1305.6006 [hep-lat]].

[141] http://www.usqcd.org/documents/13BSM.pdf, February11, 2013; T. Appelquist, R. Brower, S. Catterall, G. Fleming, J. Giedt, A. Hasenfratz, J. Kuti and E. Neil et al., arXiv:1309.1206 [hep-lat].

[142] Y. Aoki et al. [LatKMI Collaboration], Phys. Rev. D 87, no. 9, 094511 (2013) [arXiv:1302.6859 [hep-lat]].

[143] M. E. Peskin, Nucl. Phys. B 175, 197 (1980).

[144] M. Schmaltz and D. Tucker-Smith, Ann. Rev. Nucl. Part. Sci. 55, 229 (2005) [hep-ph/0502182].

[145] E. Katz, J. -y. Lee, A. E. Nelson and D. G. E. Walker, JHEP 0510, 088 (2005) [hep-ph/0312287].

[146] J. Galloway, J. A. Evans, M. A. Luty and R. A. Tacchi, JHEP 1010, 086 (2010) [arXiv:1001.1361 [hep-ph]].

[147] R. Lewis, C. Pica and F. Sannino, Phys. Rev. D 85, 014504 (2012) [arXiv:1109.3513 [hep-ph]].

[148] S. Catterall and A. Veernala, arXiv:1306.5668 [hep-lat]. 
[149] M. G. Endres, Phys. Rev. D 79, 094503 (2009) [arXiv:0902.4267 [hep-lat]].

[150] K. A. Intriligator, N. Seiberg and D. Shih, JHEP 0604, 021 (2006) [hep-th/0602239].

[151] J. Giedt, R. Brower, S. Catterall, G. T. Fleming and P. Vranas, Phys. Rev. D 79, 025015 (2009) [arXiv:0810.5746 [hep-lat]].

[152] J. W. Elliott, J. Giedt and G. D. Moore, Phys. Rev. D 78, 081701 (2008) [arXiv:0806.0013 [hep-lat]].

[153] S. Catterall, P. H. Damgaard, T. Degrand, R. Galvez and D. Mehta, JHEP 1211, 072 (2012) [arXiv:1209.5285 [hep-lat]].

[154] M. E. Peskin and T. Takeuchi, Phys. Rev. D 46, 381 (1992).

[155] M. Baak, M. Goebel, J. Haller, A. Hoecker, D. Kennedy, R. Kogler, K. Moenig and M. Schott et al., Eur. Phys. J. C 72, 2205 (2012) [arXiv:1209.2716 [hep-ph]].

[156] E. Shintani et al. [JLQCD Collaboration], Phys. Rev. Lett. 101, 242001 (2008) [arXiv:0806.4222 [hep-lat]].

[157] P. A. Boyle et al. [RBC and UKQCD Collaborations], Phys. Rev. D 81, 014504 (2010) [arXiv:0909.4931 [hep-lat]].

[158] T. Appelquist et al. [LSD Collaboration], Phys. Rev. Lett. 106, 231601 (2011) [arXiv:1009.5967 [hep-ph]].

[159] D. Schaich [LSD Collaboration], PoS LATTICE 2011, 087 (2011) [arXiv:1111.4993 [hep-lat]].

[160] T. DeGrand, arXiv:1006.3777 [hep-lat].

[161] T. Appelquist, R. Babich, R. C. Brower, M. I. Buchoff, M. Cheng, M. A. Clark, S. D. Cohen and G. T. Fleming et al., Phys. Rev. D 85, 074505 (2012) [arXiv:1201.3977 [hep-lat]].

[162] T. Appelquist and C. W. Bernard, Phys. Rev. D 22, 200 (1980).

[163] T. Appelquist and G. -H. Wu, Phys. Rev. D 48, 3235 (1993) [hep-ph/9304240].

[164] J. Bagger, V. D. Barger, K. -m. Cheung, J. F. Gunion, T. Han, G. A. Ladinsky, R. Rosenfeld and C. P. Yuan, Phys. Rev. D 49, 1246 (1994) [hep-ph/9306256].

[165] D. B. Kaplan, Phys. Rev. Lett. 68, 741 (1992).

[166] D. E. Kaplan, M. A. Luty and K. M. Zurek, Phys. Rev. D 79, 115016 (2009) [arXiv:0901.4117 [hep-ph]].

[167] R. S. Chivukula and T. P. Walker, Nucl. Phys. B 329, 445 (1990).

[168] S. B. Gudnason, C. Kouvaris and F. Sannino, Phys. Rev. D 74, 095008 (2006) [hep-ph/0608055].

[169] E. Aprile et al. [XENON100 Collaboration], Phys. Rev. Lett. 109, 181301 (2012) [arXiv:1207.5988 [astro-ph.CO]]. 